\title{
Gain-switched seed laser for Deep Space communication applications
}

\author{
Paweł Grześ, Maria Michalska, and Jacek Świderski \\ Institute of Optoelectronics, Military University of Technology, Urbanowicza 2, 00-908 Warsaw, Poland
}

Received May 31, 2018; accepted June 25, 2018; published June 30, 2018

\begin{abstract}
Deep Space (DS) communication plays an important role in space exploration programs, especially in interplanetary flights projects. To resolve the limitations of a well-known microwave link, optical communication is considered. In the article a gain-switched seed laser for a high power transmitter in the Master Oscillator Power Amplifier (MOPA) architecture is presented. This optical signal source is able to generate picosecond pulses on demand and is suitable for a high speed data link over a long range. The laser is dedicated to the pulse position modulation (PPM) scheme for low power consuming, high capacity and energy efficient optical communication.
\end{abstract}

One of the main challenges in space exploration programs is fast and reliable communication. An increasing demand on the data throughput requires more advanced technologies than well-known radio frequency (RF) techniques. To overcome the limitations of a radio link, in the 1970s the idea of optical wireless communication (OWC) was born. An optical link presents better performance, smaller payload weight and lower power consumption. Recent developments in optics and optoelectronics have led to the first successful demonstrations of long range OWC. The obtained results were satisfactory and proved that optical communication will become the next frontier for a high speed broadband connection.

The complexity of a communication system rises with the distance between a transmitter and a receiver. The most challenging task is Deep Space (DS) communication (i.e. over a distance more than 2 million kilometres from the Earth according to the ITU definition). In these systems many problems are met, such as extremely low power signal reception or high precision of the pointing system requirement. Moreover, if a terminal is mounted on a spacecraft, low mass, low volume and low power consumption requirements have to be met. All issues mentioned above are easier to solve in an optical spectrum band due to its physical properties [1].

First attempts of high speed optical communication were successfully performed only on relatively short distances (i.e. intersatellite links) [2]. In 2013 the Lunar Laser Communications Demonstration (LLCD) proved the feasibility of optical communications from beyond Earth's

\footnotetext{
*E-mail: pawel.grzes@wat.edu.pl
}

orbit to the Moon [3]. In the first interplanetary optical communication project, Mars Laser Communication Demonstration (MLCD), a 1550nm fiber laser as a light source was considered [4]. Due to its unique properties, laser technology is considered as a future medium for space communication [5].

A block diagram of the optical communication link is presented in Fig. 1. Deep Space optical communication systems have to face a list of challenges, but many mitigation techniques have been already evolved. The main source of signal power losses is Earth's atmosphere. Absorption and scattering are a result of interaction between a laser beam and gases or aerosols molecules. Absorption strongly depends on the wavelength and the $780 \div 850 \mathrm{~nm}$ and $1520 \div 1600 \mathrm{~nm}$ windows are preferred. Moreover, total attenuation rises in bad atmospheric conditions (fog, rain, snow). For these reasons, optical ground stations are located at high elevations to maximize optical channel transmission. Another source of problem is atmospheric turbulence caused by temperature and pressure variances along the signal path. It results in laser spot size and shape distortions. The atmosphere is also a source of background noise [6].

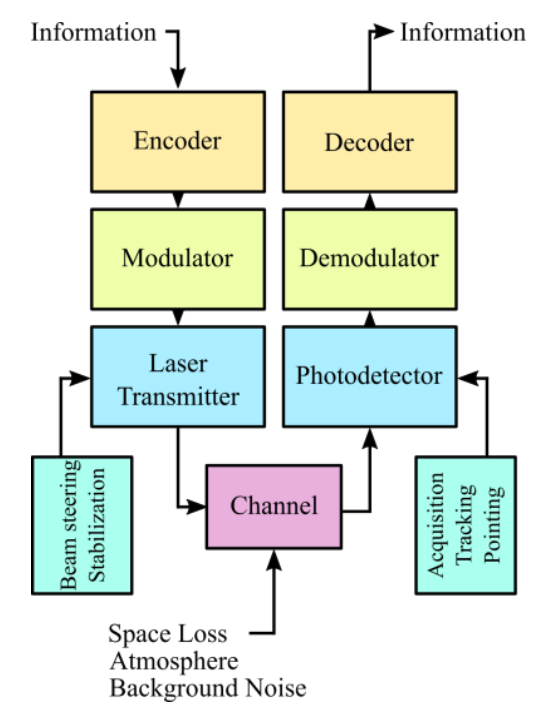

Fig. 1. Block diagram of the optical communication link. 
Due to an extremely narrow beam divergence, a high performance beam steering, acquisition, tracking and pointing (ATP) system is required. Every misalignment leads to a connection loss. It may be caused by satellite platform vibrations or mechanical stress inside the construction.

A great symbol-to-noise ratio gain can be achieved by choosing proper modulation and error correction coding (ECC) schemes. The most promising modulation method for Deep Space optical communication is pulse position modulation (PPM) and its variants (Figure 2). In this technique information is encoded as a delay between short pulses of a signal. The main advantages of this technique are high peak-to-average power ratio, high efficiency and low complexity. To enhance the transmission in error correction and detection a number of sophisticated methods were proposed, such as the Reed-Solomon code, low-density parity-check, or serially concatenated convolutional code [7].

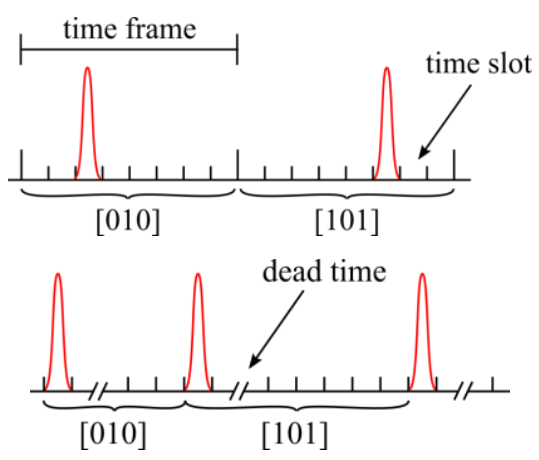

Fig. 2. PPM scheme (upper) and differential PPM (lower).

A number of optical space communication demonstrations were made and the most widely used technology in laser transmitters are solid-state bulk lasers. In recent years, fiber lasers built in the MOPA architecture have gained much interest, as a high power, efficient and reliable light source for optical communication. This setup employs a low power injection seeder (as a master oscillator) and one or several amplifying stages to provide suitable gain (Fig. 3). This topology allows to generate high power laser pulses directly controlled by electronic seed drivers.

Using a fiber-based laser system as an optical transmitter offers a number of advantages. The output pulse power is fully scalable and may be adjusted to optical link requirements. A laser beam has an excellent quality factor and reaches minimum possible divergence (diffraction limited). Pulse position modulation can be realized directly by the seed controller. The whole laser system can be made in all-fiber technology to provide integrity, high reliability and shock resistance.

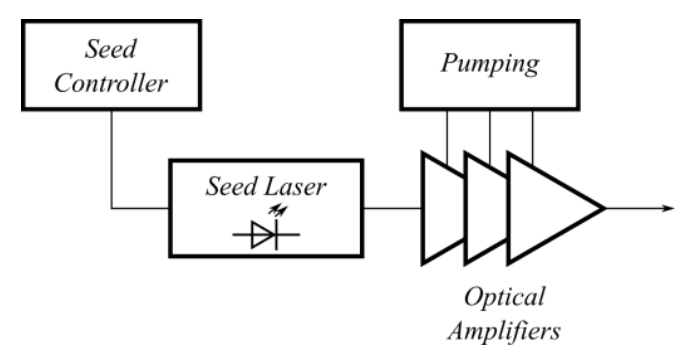

Fig. 3. Fiber laser in the MOPA topology.

In the Institute of Optoelectronics MUT a seed laser with a modulator for Deep Space optical communication has been developed. The device is based on a 1550nm distributed feedback (DFB) semiconductor laser. Applying a gain switching technique, stable 86-ps output pulses with a peak power of $1.85 \mathrm{~mW}$ have been achieved on demand. A very short optical pulse achieved allows to reduce the width of the time slot, and thus to enlarge the capacity of a transmission link. The output laser beam was only diffraction-limited with $\mathrm{M}^{2}$ parameter close to 1 , which is typical of this kind of laser sources.

The laser system operating at $1550 \mathrm{~nm}$ was chosen due to the following reasons:

- low atmosphere attenuation,

- mature technology (space grade) and availability of components,

- possibility of amplifying the signal in Er-doped and Er:Yb-doped fiber amplifiers to $\mathrm{kW}$-level peak powers,

- eye safety regulations.

In effect, the proposed seed laser can be used in the ground station transmitter as well as the spaceship communication terminal.

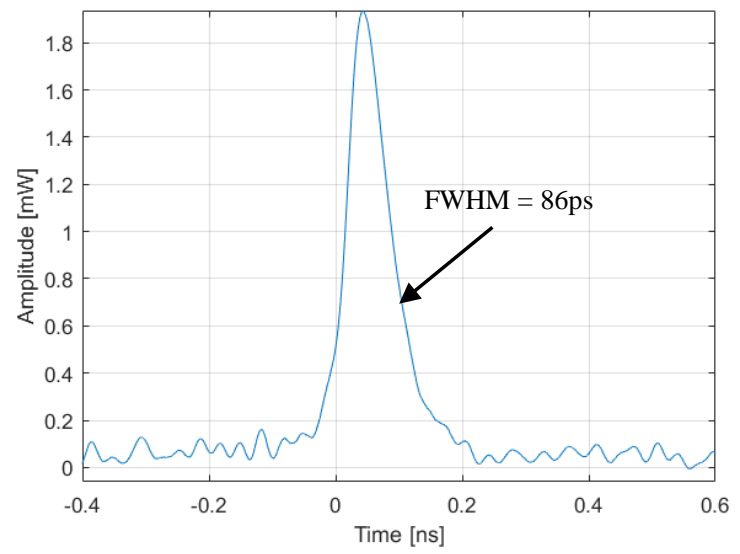

Fig. 4. Seed laser output pulse.

The differential pulse position modulator (DPPM) realized on the FPGA board defines the delay between consecutive pulses. The implemented hardware configuration based on a logic counter allows to change modulation clock frequency to increase transmission 
speed. Moreover, the dead time can be adjusted to the rest of the laser system. Additionally, an FIFO memory has been implemented to avoid data jamming. If there is no data on the input, a maintenance signal is generated.

In the article, the authors presented initial results on a gain-switched semiconductor laser and DPPM modulator dedicated to a fiber-based laser system for Deep Space communication. The wavelength of the laser is matched to the atmospheric transmission window. Picosecond output pulses allow to obtain high capacity of an optical link. The FPGA-based modulator has a fully adjustable configuration and provides a high speed signal to the laser. Additional data jamming protection and maintenance signal generation are provided. The development of a fiber laser system for Deep Space communication is planned in the near future.

The authors would like to thank Mr. Eng. Marcin Mamajek and Mr Jan Karczewski for their technical assistance. We would also like to thank AM Technologies for access to high quality test instruments. This work was partly supported by the National Science Centre, under the project No. UMO-2014/14/M/ST7/00868.

\section{References}

[1] H. Hemmati, Deep Space Optical Communications (John Wiley \& Sons 2006).

[2] T. Tolker-Nielsen, G. Oppenhauser, P. Soc. Photo-Opt. Ins. 4635, 1 (2002).

[3] D.M. Boroson et al., Proc SPIE 8971, 89710S (2014).

[4] D.M. Boroson, A. Biswas, B.L. Edwards, P. Soc. Photo-Opt. Ins. 5338, (2004).

[5] H. Hemmati, A. Biswas, I.B. Djordjevic, Proc. IEEE 99, 11 (2011).

[6] H. Kaushal, G. Kaddoum, IEEE Commun Surv Tut. 19.1, 57 (2017).

[7] B. Moision, J. Hamkins, M. Cheng, IPN Prog. Rep. 42.154, 1 (2005). 